\title{
Relação entre condições climáticas e incidência de dengue no município de Campo Grande, MS
}

\section{Relationship between climate and dengue incidence in the city of Campo Grande, MS}

Relación entre las condiciones del tiempo y la incidencia del dengue en Campo Grande, MS

lael Cristina da Silva Pacheco ${ }^{1}$ Alexandra Maria Almeida Carvalho Elenir Rose Jardim Cury Pontes ${ }^{3}$ Maria da Graça da Silva ${ }^{4}$

${ }^{1}$ Enfermeira, Mestre em Ciências da Saúde/UFMS (2013), Diretora do Centro Universitário Anhanguera de Campo Grande- Unidade 2. E-mail: iaell@hotmail.com

${ }^{2}$ Engenheira, Doutora em Biosystems Eng. and Environmental Science pela The University of Tennessee, Estados Unidos (2001), Professora Adjunta 2 da Universidade Federal de Mato Grosso do Sul. E-mail: amjojv@gmail.com

${ }^{3}$ Odontóloga, Doutora em Saúde Pública pela Universidade de São Paulo (2002), Professor adjunto da Universidade Federal de Mato Grosso do Sul.

E-mail: elenirpontes@uol.com.br

${ }^{4}$ Enfermeira, Doutora em Enfermagem pela Escola de Enfermagem de Ribeirão Preto - USP, docente da Universidade Federal de Mato Grosso do Sul (UFMS). E-mail: gracasilva51@gmail.com 
Resumo: As mudanças climáticas têm sido observadas pela população mundial, e seus fatores podem afetar o ciclo biológico de diversas doenças, como a dengue, enfermidade de caráter endêmico/epidêmico, transmitida pelo Aedes agyptis. Diante disso, o objetivo deste estudo foi relacionar incidência de dengue com alterações climáticas no município de Campo Grande, MS, Brasil, no período 2001-2012. Os resultados indicam que há relação entre os casos confirmados de dengue e a temperatura do ar (média mensal $\left({ }^{\circ} \mathrm{C}\right)$, média mínima mensal $\left({ }^{\circ} \mathrm{C}\right)$, precipitação (quantidade total - valor mensal $(\mathrm{mm})$ ) e umidade relativa do ar (média mensal (\%)); não evidenciando relação estatística significante com a temperatura média máxima mensal $\left(35,5^{\circ} \mathrm{C}\right)$. De julho a outubro, a taxa de incidência de dengue por 100 mil habitantes foi baixa, elevando-se nos meses de novembro a março, quando ocorrem valores medianos mensais maiores de temperatura do ar, temperatura mínima, umidade relativa do ar e precipitação mensal.

Palavras-chave: clima; mudança climática; atenção à saúde; febre da dengue.

Abstract: Climate change has been observed by the world population and its factors can affect the biological cycle of several diseases, such as dengue, an endemic / epidemic disease, transmitted by Aedes agyptis. Therefore, the objective of this study was to correlate the incidence of dengue with climate change in the municipality of Campo Grande, MS, Brazil, in the period 2001-2012. The results indicate that there is a relationship between confirmed dengue cases and air temperature (monthly average $\left({ }^{\circ} \mathrm{C}\right.$ ), monthly minimum $\left({ }^{\circ} \mathrm{C}\right)$, precipitation (total amount- monthly value $(\mathrm{mm})$ ) and relative humidity; not showing a statistically significant relationship with the mean maximum monthly temperature $\left(35.5^{\circ} \mathrm{C}\right)$. From July to October, the incidence of dengue fever per 100,000 inhabitants was low, rising in the months of November to March, where higher monthly mean values of air temperature, minimum temperature, relative humidity and monthly precipitation occur.

Key words: climate; climate change; health care; dengue.

Resumen: El cambio climático ha sido observado por la población del mundo y sus factores puede afectar el ciclo de vida de diversas enfermedades, como el dengue, enfermedad de carácter endémico/ epidémic, transmitidas por el Aedes agyptis. Por lo tanto, el objetivo de este estudio fue correlacionar la incidencia de la fiebre del dengue con el cambio climático en la ciudad de Campo Grande, MS, Brasil, en el período 2001-2012. Los resultados indican que existe una relación entre los casos confirmados de dengue y la temperatura del aire (promedio mensual $\left({ }^{\circ} \mathrm{C}\right.$ ), la media mensual mínima $\left({ }^{\circ} \mathrm{C}\right)$, la precipitación (cantidad total-mensual $(\mathrm{mm})$ ) y humedad relativa (promedio mensual (\%)), peo non muestra una relación estadísticamente significativa con la temperatura máxima media mensual $\left(35,5^{\circ} \mathrm{C}\right)$. De julio a octubre, la tasa de incidencia del dengue por cada 100 mil habitantes fue baja, el aumento en noviembre. a marzo, donde hay valores de la mediana mensuales más altos de temperatura del aire, la temperatura mínima, la humedad relativa y la precipitación mensual.

Palabras clave: clima; cambio climático; atención a la salud; fiebre del dengue. 


\section{INTRODUÇÃO}

A Variabilidade Climática é entendida como uma propriedade intrínseca do sistema climático terrestre, responsável por oscilações naturais nos padrões climáticos, observados em nível local, regional e global. A mudança climática é uma das grandes ameaças globais do século 21 (COSTELLO et al., 2009) e pode ser explicada como uma consequência do aumento da temperatura global devido à emissão de gases poluentes resultantes das atividades antropogênicas associada com a variabilidade natural (CONFALONIERI, 2003).

Dentre os vários fatores relacionados com as mudanças climáticas, podemos citar as principais alterações de: temperatura, precipitações, umidade relativa, na biodiversidade, na agricultura, nos regimes hídricos, no aumento na emissão de gases poluentes (CO2, SO2, CH4), afetando as condições de saúde (CONRADO et al., 2010).

Nas últimas décadas, as mudanças climáticas estão sendo observadas com facilidade pela população mundial, pois as estações do ano estão sofrendo uma variabilidade climática constante. A temperatura global até o ano de 2100 poderá sofrer um acréscimo de $1,8^{\circ} \mathrm{C}$ e $4^{\circ} \mathrm{C}$, resultando em um impacto direto e indireto na saúde da população (GRUPO INTERGUBERNAMENTAL DE EXPERTOS SOBRE EL CAMBIO CLIMÁTICO [IPCC], 2007).

As modificações climáticas podem produzir impactos sobre a saúde humana por diferentes vias, de forma direta, como no caso das ondas de calor, ou mortes causadas por outros eventos extremos, como furacões e inundações (ORGANIZAÇÃO PAN-AMERICANA DA SAÚDE [OPAS], 2008). Muitas vezes, esse impacto é indireto, sendo influenciado por transformações no ambiente, como a alteração de ecossistemas e de ciclos biogeoquímicos, que podem aumentar a incidência de doenças infecciosas, mas também de agravos não transmissíveis, que incluem a desnutrição e transtornos mentais (IPCC, 2008). 
Existe um crescente interesse científico sobre a saúde da população e o potencial efeito das mudanças climáticas. Uma área que tem recebido atenção especial é a associação entre a variação do clima e doenças transmitidas por vetores (HALES et al., 2012). Dentre os vetores influenciados pelas mudanças climáticas, o Aedes aegypti está entre os mais susceptíveis, pois as condições atmosféricas, a temperatura, umidade relativa do ar e precipitação contribuem para o desenvolvimento do vetor e consequentemente na incidência da dengue (YOKOO, 2010). Tal enfermidade afeta grande parte da população urbana e suburbana, tornando-se um dos principais problemas de saúde pública internacional, possuindo um padrão de proliferação em regiões tropicais e subtropicais, com característica do clima quente e úmido (WORLD HEALTH ORGANIZATION [WHO], 2000).

As epidemias e surtos da doença ocorrem devido a fatores macro e micro determinantes (QUINTANILHA, 2010). Os macrodeterminantes são: elevadas temperatura e umidade relativa do ar; alta densidade populacional; coleta de resíduos sólidos domiciliares e abastecimento de água potável deficientes. Como microdeterminantes: percentual de susceptiveis aos sorotipos circulantes; abundância e tipos de criadouros do mosquito transmissor; altos índices de infestação predial; densidade de fêmeas do vetor (QUINTANILHA, 2010). À medida que aparecem mais evidências entre as alterações ambientais e a saúde humana, aumentam as possibilidades de que vetores ampliem suas áreas de proliferação (LIMA; MORAES; PEREIRA, 2011).

Por isso, este estudo teve como objetivo principal relacionar incidência de dengue com alterações climáticas no município de Campo Grande, MS, Brasil, no período 2001-2012. 


\section{MATERIAIS E MÉTODO}

Trata-se de um estudo ecológico, de série temporal, analítico, empregado para avaliar como o contexto social e ambiental pode afetar a saúde dos grupos populacionais.

Todos os casos confirmados de dengue da população residente em Campo Grande, MS, no período de 2001 a 2012, e notificados no Sistema de Informação de Agravos de Notificação (SINAN) foram analisados.

As variáveis adotadas foram: População residente do município de Campo Grande, MS; Casos de dengue: número de casos confirmados de dengue na população residente em Campo Grande, MS, e os dados climatológicos: Valor médio mensal da temperatura do $\operatorname{ar}\left({ }^{\circ} \mathrm{C}\right)$ (média, mínima e máxima); Umidade relativa do ar (\%) e Precipitação acumulada no mês ( $\mathrm{mm}$ ), obtidos mensalmente, referentes ao período de 2001 a 2012.

As variáveis referentes à população residente em Campo Grande, MS, e casos confirmados foram obtidos do DATASUS, que disponibiliza informações para tabulação pelo programa TabNe, porém os dados são provenientes do Instituto Brasileiro de Geografia e Estatística (IBGE), sendo que os de 2010 provêm ao Censo Demográfico e os de 2001 a 2009, 2011 e 2012 tratam-se de estimativas populacionais.

A fonte de dados da variável dengue foi o SINAN. Esse sistema é alimentado pelas notificações e investigação de casos de doenças e agravos que constam na lista nacional de doenças de notificação. Os casos são notificados em formulário próprio e posteriormente inseridos no sistema pelos profissionais de saúde.

O número de casos novos confirmado, clássico e febre hemorrágica da dengue (códigos A90-A91 da CID-10), por 100 mil habitantes, na população residente em determinado espaço geográfico, no ano considerado, é o indicador Taxa de Incidência de Dengue-D.2.3-2011, 
calculado pela equação: Taxa de incidência de dengue = № de casos de dengue confirmados em residentes/ População total residente $X$ 100.000.

Para calcular esse indicador, foram tabulados no TabNet os casos confirmados para residentes em Campo Grande, MS, segundo o mês dos primeiro sintomas, para os anos 2001-2012. As tabelas geradas online, via TabNet, foram salvas em arquivos CSV (valores separados por vírgula), posteriormente organizados na base de dados da pesquisa, utilizando o Excel.

Os dados meteorológicos (temperatura, precipitação e umidade relativa do ar) foram retirados da base de dados online do Instituto de Controle do Espaço Aéreo (ICEA) sob a responsabilidade de Subdivisão de Climatologia Aeronáutica da Divisão de Pesquisa e Desenvolvimento (ICEA, s.d.). As variáveis climatológicas foram selecionadas para o período de estudo e os resultados gerados apresentados na forma de relatórios no site do instituto. Os parâmetros temperatura do $\operatorname{ar}\left({ }^{\circ} \mathrm{C}\right) \mathrm{e}$ umidade relativa (\%) se referem à média mensal dos valores unitários presentes no Banco de Dados Climatológicos do ICEA. O parâmetro precipitação mensal $(\mathrm{mm})$ representa a precipitação acumulada de cada mês.

O comportamento anual da incidência da doença em Campo Grande nos anos estudados originou os dois períodos distintos, chamados neste estudo de baixa temporada (julho a outubro), e alta temporada (novembro a junho). Essa classificação foi utilizada para selecionar os valores das variáveis climáticas referentes a cada período. Para investigar a hipótese de que a incidência de dengue depende das variáveis climáticas, foi utilizado o teste de Mann-Whitney, no programa Bioestat.

Este trabalho foi aprovado pelo Comitê de Ética em Pesquisa vinculado à Universidade Federal de Mato Grosso do Sul, parecer n. 87566 e Certificado de Apresentação para Apreciação Ética (CAAE) 
registro n. 04885312.4.0000.0021. Tratando-se de um estudo com dados secundários, foi aprovada a dispensa do Termo de Consentimento Livre e Esclarecido (TCLE), assumiu-se o compromisso de atender os requisitos éticos necessários. Os dados meteorológicos foram autorizados pelo Departamento de Controle Aéreo.

\section{RESULTADOS}

O comportamento anual da taxa de incidência de dengue por 100.000 habitantes para Campo Grande, MS, para os anos de 2001 e 2012, é mostrado na Figura 1. De julho a outubro a taxa de incidência por 100 mil habitantes é baixa $(4,1 \pm 5,7)$, e, nos outros meses, a incidência aumenta consideravelmente $(74,8 \pm 177,2)$.

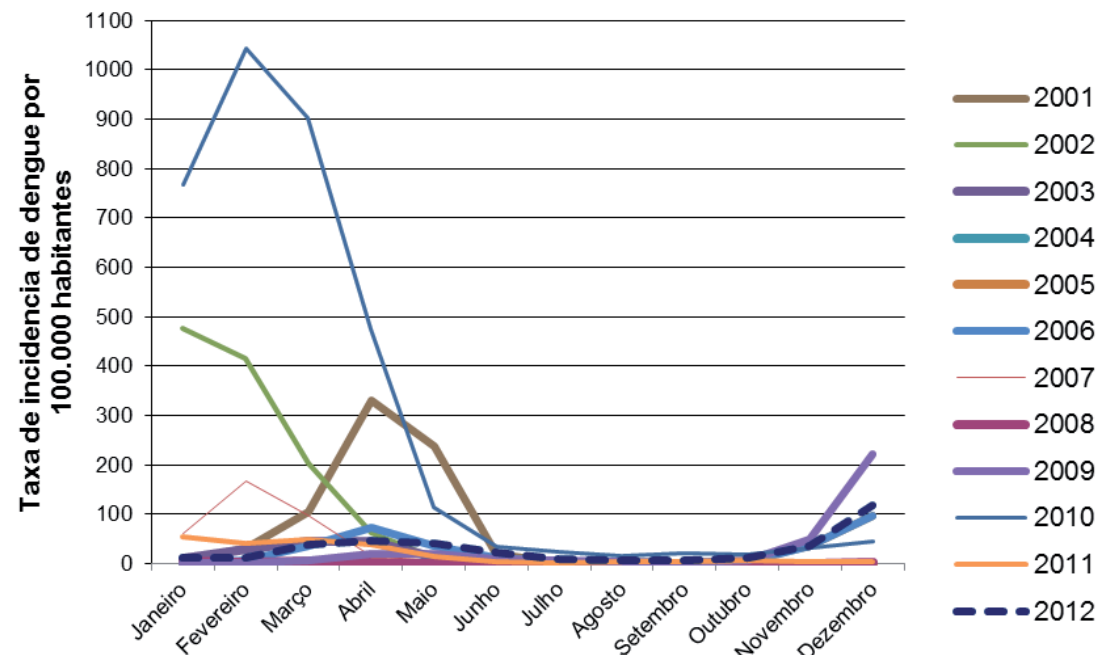

Figura 1 - Taxa de incidência de dengue por 100 mil habitantes. Campo Grande, MS, 2001-2012 
A incidência da dengue nos anos de 2001, 2002, 2007 e 2010 é considerada como uma incidência alta para o número de habitantes (Figura 1), sendo mais predominantes as notificações nos meses de janeiro a julho, porém com início moderado nos meses de novembro e dezembro.

A caracterização climática de Campo Grande foi estudada pelas seguintes variáveis: temperatura $\left({ }^{\circ} \mathrm{C}\right)$, precipitação acumulada $(\mathrm{mm})$ e umidade relativa (\%). A Figura 2 mostra, no mesmo gráfico, a taxa de incidência de dengue por 100.000 habitantes e a temperatura do ar mensal $\left({ }^{\circ} \mathrm{C}\right)$ (média, máxima e mínima), de 2001 a 2012. Gráficos similares para umidade relativa mensal (\%) e precipitação acumulada mensal (mm) são mostrados nas Figuras 3 e 4, respectivamente.

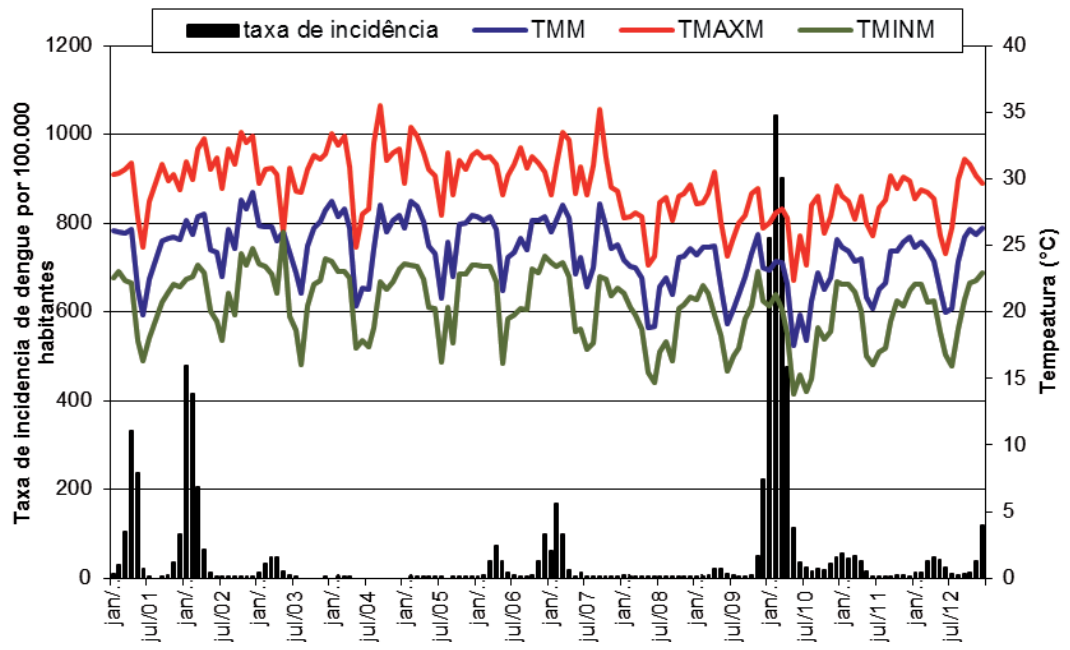

Figura 2 - Taxa de incidência de dengue por 100 mil habitantes e temperatura mensal: média, máxima e mínima $\left({ }^{\circ} \mathrm{C}\right)$. Campo Grande, MS, 2001-2012

TMM $=$ Temperatura do Ar- Média Mensal $\left({ }^{\circ} \mathrm{C}\right)$.

TMAXM = Temperatura do Ar- Valor Médio Máximo Mensal $\left({ }^{\circ} \mathrm{C}\right)$.

TMINM = Temperatura do Ar- Valor Médio Mínimo Mensal $\left({ }^{\circ} \mathrm{C}\right)$. 


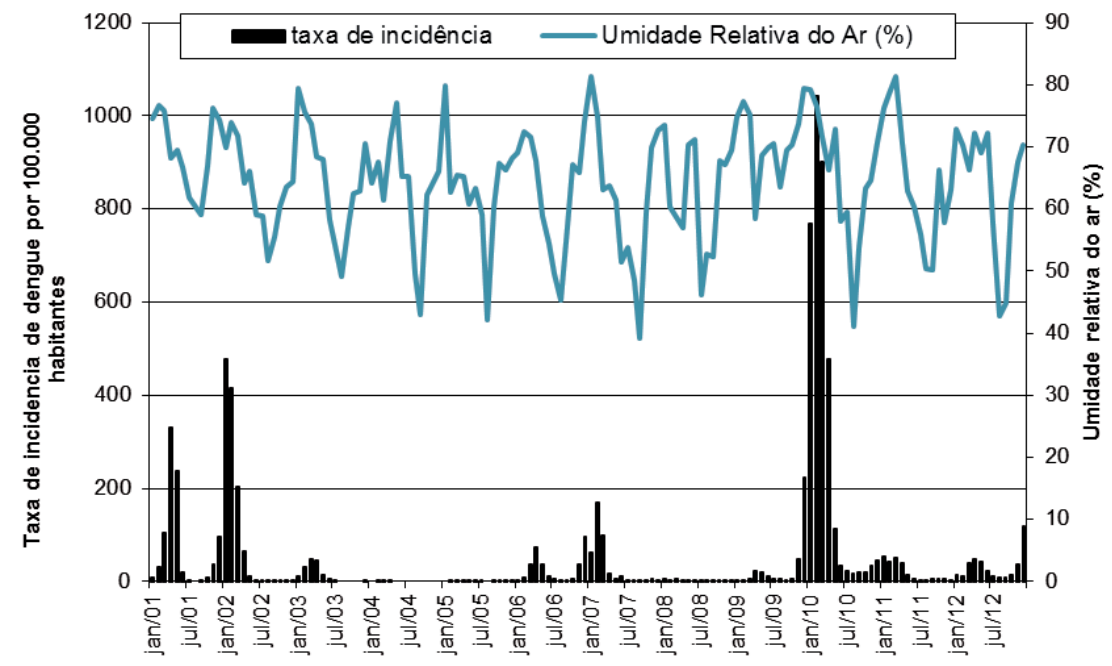

Figura 3 - Taxa de incidência de dengue por 100 mil habitantes e umidade relativa do ar-média mensal (\%). Campo Grande, MS, 2001-2012

Os dados climatológicos foram comparados para os dois grupos, temporada de alta e de baixa incidência de dengue, e os resultados estão apresentados na Tabela 1. Ao comparar as variáveis climáticas para os dois grupos, apenas não houve diferença estatisticamente significativa para a temperatura máxima mensal, que foi mais baixa no período de alta incidência. A temperatura ideal para ciclo biológico do mosquito é de $22^{\circ} \mathrm{C}$ e $30^{\circ} \mathrm{C}$, assim é favorável para o desenvolvimento nas temperaturas quentes, porém não extremamente quentes, pois as faixas altas interferem na vida do Aedes aegypti. 
Tabela 1- Comparação entre os grupos taxa de incidência de dengue por 100 mil habitantes e as variáveis climáticas. Campo Grande, MS, janeiro de 2001 a dezembro de 2012

\begin{tabular}{lrrrrrrrrr}
\hline \multirow{2}{*}{ Variáveis } & \multicolumn{6}{c}{ Taxa de incidência/100 mil habitantes } & \multirow{2}{*}{$\boldsymbol{p}$} \\
\cline { 2 - 9 } & \multicolumn{1}{c}{ Méd } & \multicolumn{1}{c}{ DP } & \multicolumn{1}{c}{ Mín } & \multicolumn{1}{c}{ Máx } & Méd. & DP & Mín & Máx & \\
\hline TMM & 24,2 & 2,2 & 17,43 & 28,9 & 23,4 & 2,5 & 17,9 & 28,4 & $<0,001$ \\
TMINM & 21,6 & 2,2 & 13,8 & 26,0 & 18,9 & 2,4 & 14,0 & 24,3 & $<0,001$ \\
TMAXM & 29,7 & 2,3 & 22,4 & 33,8 & 29,3 & 2,7 & 23,5 & 35,5 & 0,375 \\
PRECM & 144,8 & 82,2 & 0 & 391,2 & 74,1 & 64,6 & 0 & 218,8 & $<0,001$ \\
URM & 69,5 & 5,6 & 56,9 & 81,4 & 57,6 & 8,4 & 39,2 & 72,2 & $<0,001$ \\
\hline
\end{tabular}

Nota: Teste de Mann-Withney

Méd = média; DP = Desvio padrão; Mín = valor mínimo; Máx = valor máximo.

$\mathrm{TMM}=$ Temperatura do Ar- Média Mensal $\left({ }^{\circ} \mathrm{C}\right)$.

TMAXM = Temperatura do Ar- Valor Médio Máximo Mensal $\left({ }^{\circ} \mathrm{C}\right)$.

TMINM = Temperatura do Ar- Valor Médio Mínimo Mensal $\left({ }^{\circ} \mathrm{C}\right)$.

PRECM = Precipitação- Quantidade Total-Valor Mensal (mm).

URM = Umidade Relativa do Ar- Média Mensal (\%).

Quanto à relação da taxa de incidência e precipitação, houve picos em particular nos anos de 2001, 2002, 2007 e 2010, após os períodos de chuva (Figura 4).

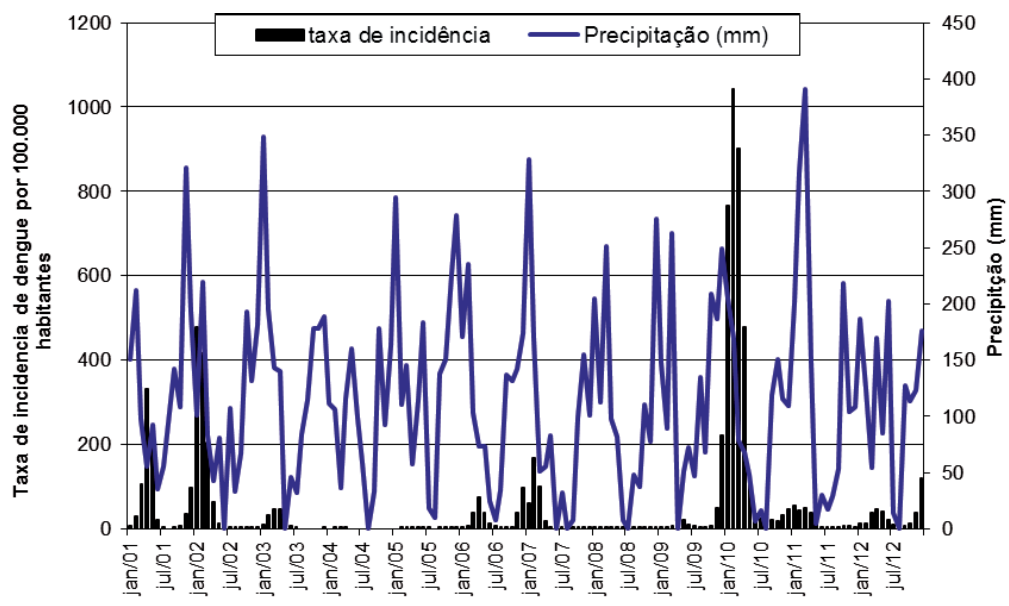

Figura 4 - Taxa de incidência de dengue por 100 mil habitantes e precipitação acumulada mensal (mm). Campo Grande, MS, 2001-2012 


\section{DISCUSSÃO}

Considerando os resultados encontrados, percebemos que outros estudos (FIGUEIREDO et al., 2004; CÂMARA; MATOS, 2007) apontam a mesma tendência, os quais mostram que os cinco primeiros meses do ano correspondem ao período mais quente e úmido, característico para proliferação do mosquito e aumento de casos de dengue, padrão correspondente aos casos notificados no Brasil. No Município de Campo Grande, MS, as temperaturas médias variaram de $20^{\circ} \mathrm{C}$ (mínima) e $29^{\circ} \mathrm{C}$ (máxima), no período de 2001 a 2012.

Na revisão sistemática de Viana e Ignotti (2013), cujo objetivo foi pesquisar a literatura científica sobre a ocorrência da dengue no Brasil e sua relação com variáveis meteorológicas, os autores constataram relação entre incidência da dengue com a temperatura e pluviosidade, sendo a associação mais expressiva a partir do segundo até o quarto mês do ano, e concluíram que a dengue está fortemente relacionada com variáveis meteorológicas.

Um estudo realizado sobre a biologia e as exigências térmicas do Aedes aegypti, testou o ciclo de vida em cinco temperaturas médias $18^{\circ} \mathrm{C}, 22^{\circ} \mathrm{C}, 26^{\circ} \mathrm{C}, 30^{\circ} \mathrm{C}$ e $34^{\circ} \mathrm{C}$. Como resultado, foi comprovada uma interação significativa no ciclo biológico do mosquito quando exposto a diferentes temperaturas, sendo favorável para o desenvolvimento a temperatura de $22^{\circ} \mathrm{C}$ e $30^{\circ} \mathrm{C}$, e que os extremos de temperatura de $18^{\circ} \mathrm{C}$ e $34^{\circ} \mathrm{C}$ apresentaram efeitos negativos. Portanto, considerando os dados climatológicos de quatro regiões de estudo do estado da Paraíba, afirma-se que os municípios apresentaram condições favoráveis ao estabelecimento da população do vetor durante todo o ano (FERNANDES et al., 2006).

Nos períodos de temperatura baixa, existe uma queda considerável nos casos confirmados da doença, porém o vírus não deixa de circular na população. Porém, nos meses considerados quentes, 
há uma elevação da temperatura, dos índices pluviométricos e da umidade relativa do ar, fatores ambientais altamente propícios à proliferação do vetor da dengue (CASALI et al., 2004).

Neste estudo, podemos verificar que os picos de umidade relativa do ar coincidem com os picos de incidência de dengue na população, nos anos com maior taxa de incidência (Figura 3). A umidade relativa influencia na longevidade do vetor, beneficiando a fêmea do mosquito a completar mais de um ciclo de replicação do vírus (DONALÍSIO; GLASSER, 2002). Os achados coincidem com resultados similares do trabalho realizado entre 2008 a 2010, em Tangará da Serra, MT, onde os períodos de maior umidade foram dezembro, janeiro, fevereiro, março e abril. $\mathrm{O}$ ano que apresentou os valores mais elevados foi 2009, que atingiu os maiores casos da doença (FERNANDES et at., 2012). A longevidade do mosquito adulto varia, podendo manter-se a $10^{\circ} \mathrm{C}$ e com $100 \%$ de umidade relativa do ar, vivendo por 30 dias, porém, sem alimentos e submetidos a $23^{\circ} \mathrm{C}$, com $70 \%$ de umidade, vivem somente quatro dias (TORRES, 2005).

Um estudo comparativo das relações climáticas com a incidência da doença em Campo Grande, MS, no ano 2009 e 2010, comprovou, na análise dos dados, que em 2010, o aparecimento de áreas maiores inundadas, o crescimento da vegetação e a intensificação da pluviosidade contribuíram para o aumento da taxa de incidência (LIMA; MORAES; PEREIRA, 2011).

De acordo com estudos realizados na região nordeste, o elevado índice pluviométrico associado com acréscimo da temperatura, causou o aumento populacional do mosquito e, consequentemente, o número de casos confirmados da doença. A pluviosidade não só aumentou consideravelmente a quantidade de criadouros disponíveis para o desenvolvimento das formas imaturas do vetor, como também gerou condições ambientais mais apropriadas para o desenvolvimento de mosquitos adultos (SANTOS; LEITE, 2011), mas diferente da região 
centro- oeste, os meses destacados foram maio, junho e julho (LIMA; MOREIRA; NÓBREGA, 2016).

Furtado, Souza e Aguiar (2011), em pesquisa realizada na Amazônia Ocidental, para avaliar a influência de elementos micrometeorológicos nos casos de dengue no município de Ji-Paraná, Rondônia, nos anos de 2002 a 2008, reforçam que os fatores naturais influenciam no processo de proliferação da dengue, com destaque para o clima - pois a incidência dessa doença varia de acordo com as condições climáticas, sendo a precipitação, a umidade do ar e a radiação solar, as condições mais favoráveis. No referido estudo, foi verificado que a precipitação é o fator que mais influencia a incidência da dengue, embora não tenha havido valores estatísticos significativos.

Em estudo semelhante ao presente, tendo como objetivo verificar a relação entre a incidência de dengue e as alterações climáticas no município de Cascavel, PR, entre os anos de 2007 a 2011, ficou evidente que os casos aparecem em maior número no período de janeiro a maio, o que coincidiu com o período de maior volume de chuvas. Além disso, nos meses que apresentaram temperaturas entre $21^{\circ} \mathrm{C}$ e $29^{\circ} \mathrm{C}$ (fevereiro, março e abril) e umidade relativa superior a $70 \%$, houve aumento na proliferação do mosquito, indicando correlação positiva entre o número de casos notificados, a temperatura e o volume de chuva. Os autores pontuam que as condições climáticas não são causa exclusiva da dengue, mas estão relacionadas com a doença por estarem diretamente ligadas ao ciclo de desenvolvimento do vetor (ANTONIO; PEDER; SILVA, 2015).

Outro estudo, cujo objetivo foi investigar os fatores climáticos associados aos de casos da dengue na cidade de João Pessoa, Paraíba, no período de 2007 a 2011, constatou que a precipitação e a umidade relativa do ar são as variáveis climáticas que mais favorecem a ocorrência de dengue (SILVA et al. 2015). 
Apesar do ciclo biológico do Aedes aegypti ser conhecido na dengue, Pignatti (2004) afirma que o vetor da doença encontra no ambiente urbano, as condições ideais para se procriar e desencadear surtos da doença, associados ao aumento da produção de materiais descartáveis, à negligência com o lixo e ao aumento de recipientes que acumulam água nos domicílios humanos, além da densidade populacional.

Ressaltamos que esta é uma questão mundial, sendo necessário um conjunto de ações, trabalho multidisciplinar e multissetorial, com a interação entre profissionais das áreas de saúde, clima e geografia, que poderiam assessorar os setores da saúde a compreender a transmissão dessa enfermidade, adoção de medidas preventivas de vetores, com atuação predominante das equipes de saúde, envolvendo agentes comunitários de saúde e agentes de controle de endemias e o profissional de enfermagem da atenção básica, com a orientação e monitoramento do enfermeiro, visando promover mudanças de hábitos da comunidade para tornar os domicílios livres do Aedes aegypti.

\section{CONCLUSÃO}

O município investigado apresenta casos de dengue durante todo o ano, diminuindo a incidência somente durante quatro meses do ano, de julho a outubro. As séries históricas mostraram que os períodos de alta incidência da doença ocorreram nas faixas de temperatura ideais para o mosquito (quente, mas não muito elevada), com alta umidade relativa do ar, logo após as épocas com maiores valores de precipitação. Essas condições de clima da cidade favorecem a incidência de dengue e estão relacionadas à ocorrência de endemias e epidemias.

Elaboração de ferramentas preditivas, para análise das diferentes variáveis climatológicas em intervalos de tempo pode subsidiar 
o entendimento da dinâmica do Aedes aegypti nos municípios, estados e países, auxiliando nas ações de monitoramento e combate ao mosquito transmissor.

Além desses fatores, o comportamento das pessoas influencia na proliferação do vetor, essencialmente nos períodos sazonais secos (baixa incidência) para transmissão, negligenciando os cuidados preventivos, pois os locais mais propícios para o criadouro são os ambientes domésticos. Assim sendo, é fundamental que o controle desses locais seja constantemente monitorado pela atenção básica e pelos programas de conscientização do combate a dengue.

O município de Campo Grande, MS, através da Secretaria Municipal de Saúde (SESAU), possui o comitê de mobilização de combate à dengue, tendo como objetivo a mobilização para educação permanente da população, através de parcerias com órgão e entidades, como o Conselho Regional de Engenharia, Arquitetura e Agronomia (CREA/MS), conscientização nas escolas, informes educativos no jornal da Associação Comercial e a vistoria em imóveis que estão para alugar e/ou vender (SESAU, 2011).

Para que essas ações sejam efetivas, os governantes precisam refletir antecipadamente sobre um planejamento financeiro para melhoria dos serviços de saúde oferecidos para a sociedade e profissionais de saúde capacitados para o atendimento da população, minimizando os agravos na saúde decorrentes das mudanças climáticas.

\section{REFERÊNCIAS}

ANTONIO, G.; PEDER, L. D. de; SILVA, C. M. da. Influência das alterações climáticas nos casos de dengue nos anos de 2007 A 2011 no município de Cascavel - PR. SaBios - Revista de Saúde e Biologia, v. 10, n. 1, p. 06-14, jan./abr. 2015.

CÂMARA, D. C. P.; MATOS, R. R. C. Estudo retrospectivo (histórico) da dengue no Brasil: características regionais e dinâmicas. Revista da Sociedade 
Brasileira de Medicina Tropical, Uberaba, MG, v. 40, n. 2, p. 192-196, mar./ abr. 2007.

CASALI, C. G. et al. A epidemia de dengue/dengue hemorrágico no município do Rio de Janeiro, 2001/2002. Revista Sociedade Brasileira de Medicina Tropical, Uberaba, MG, v. 37, n. 4, p. 296-9, jul./ago. 2004.

CONFALONIERI, U. E. C. Variabilidade climática, vulnerabilidade social e saúde no Brasil. Terra Livre, São Paulo, ano 19, v. I, n. 20, p. 193-204, jan./jun. 2003.

CONRADO, D. et al. Vulnerabilidades e as mudanças climáticas. Rio de Janeiro: IIEB, 2000. Disponível em: <www.bvsde.paho.org/bvsacd/cd68/ DConrado.pdf>. Acesso em: 23 ago. 2010.

COSTELLO, A. et al. Managing the health effects of climate change. The Lancet, v. 373, n. 9676, p. 1693-1733, maio 2009.

DONALÍSIO, M. R.; GLASSER, C. M. Vigilância entomológica e controle de vetores do dengue. Revista Brasileira de Epidemiologia, São Paulo, v. 5, n. 3, p. 259-272, 2002.

FERNANDES, R. S. et al. Clima e casos de dengue em Tangará da Serra/MT. Hygeia, v. 8, n. 15, p. 78-88, dez. 2012.

FERNANDES, C. et al. Biologia e exigências térmicas de Aedes aegypti (L.) (Diptera: Culicidae) provenientes de quatro regiões bioclimáticas da Paraíba. Neotroprical Entomology, v. 35, n. 6, p. 853-860, nov./dez. 2006.

FIGUEIREDO, R. M. P. et al. Doenças exantemáticas e primeira epidemia de dengue ocorrida em Manaus, Amazonas, no período de 1998-1999. Revista da Sociedade Brasileira de Medicina Tropical, Uberaba, MG, v. 37, n. 6, p. 476-479, nov./dez. 2004.

FURTADO, R. da S.; SOUZA V. A. de; AGUIAR, R. G. A influência de elementos micrometeorológicos na incidência dos casos de dengue na Amazônia ocidental. In: SEABRA, Giovanni; MENDONÇA, Ivo (Org.). Educação ambiental: responsabilidade para a conservação da sociobiodiversidade. João Pessoa, PB: Editora da UFPB, 2011.

GRUPO INTERGUBERNAMENTAL DE EXPERTOS SOBRE EL CAMBIO CLIMÁTICO (IPCC). Cambio climático 2007: informe de sínteses. Genebra, Suíça: IPCC, 2008. Disponível em: <https://www.ipcc.ch/pdf/assessment-report/ar4/ syr/ar4_syr_sp.pdf>. 
Relação entre condições climáticas e incidência de dengue no município de Campo Grande, MS

HALES, S. et al. Potential effect of population and climate changes on global distribution of dengue fever: an empirical model. The Lancet, v. 360, n. 9336, p. 830-834, set. 2002. Disponível em: <http://image.thelancet.com/ extras/01art11175web.pdf>. Acesso em: 5 jun. 2012.

INSTITUTO DE CONTROLE DO ESPAÇO AÉREO (ICEA). Banco de Dados Climatológicos do Comando da Aeronáutica. [s.d.]. Disponível em: <http:// clima.icea.gov.br/clima/>. Acesso em: 6 fev. 2013.

LIMA, S. F. S.; MORAES, E. C.; PEREIRA, G. Análise das relações entre as variáveis ambientais e a incidência de dengue no município de Campo Grande-MS. In: SIMPÓSIO BRASILEIRO DE SENSORIAMENTO REMOTO SBSR, 15. Curitiba, PR, 30 de abril a 5 de maio de 2011. Anais... Curitiba, 2011. Disponível em: <http://www.dsr.inpe.br/sbsr2011/files/p1250.pdf>.

LIMA, R. C. de; MOREIRA, E. B M.; NÓBREGA, R. S. A influência climática sobre a epidemia dengue na cidade do Recife por Sistema de Informações Geográficas. Revista Brasileira de Geografia Física, v. 9, n. 2, p. 384-398, 2016. Disponível em: <http://www.revista.ufpe.br/rbgfe/index.php/revista/ article/view/1398/930>.

BRASIL. Ministério da Saúde. DATASUS. TabNet 2.7: Aplicativo para realização de tabulações na Intranet/Internet. 2013. Disponível em: <ftp://ftp.datasus. gov.br/tabnet/doc/ManualTabnet.pdf>. Acesso em: 1ㅇ dez. 2013.

. DATASUS. Taxa de incidência de dengue- D.2.3 - 2011. Disponível em: <http://www.ripsa.org.br/fichasIDB/record.php?node=D.2.3\&lang=pt \&version=ed6>. Acesso em: 6 dez. 2013.

ORGANIZAÇÃO PAN-AMERICANA DA SAÚDE (OPAS). Mudanças climáticas e ambientais e seus efeitos na saúde: cenários e incertezas para o Brasil. Brasília: OPAS, 2008. Disponível em: <http://www.fiocruz.br/omsambiental/ media/Mudanca_climatica_saude1.pdf $>$.

PIGNATTI, M. G. Saúde e ambiente: as doenças emergentes no Brasil. Ambiente e Sociedade, v. 7, n. 1, p. 133-147, já./jun. 2004.

QUINTANILHA, A. C. F. Caracterização clínica e epidemiológica de casos de dengue internados em hospital público de Campo Grande - MS. 2010. Dissertação (Mestrado em Saúde e Desenvolvimento da Região CentroOeste)- Universidade Federal do Mato Grosso do Sul, Campo Grande, MS, 2010. 
SANTOS, C. L. L.; LEITE, R. F. C. Ceará: situação da dengue às margens das mudanças climáticas. In: SIMPÓSIO INTERNACIONAL DE CLIMATOLOGIA, 4. Anais... João Pessoa, PB, 2011. Disponível em: <http://www.sbmet.org.br/ sic2011/arq/74193820566027419382056.pdf>.

SECRETARIA MUNICIPAL DE SAÚDE (SESAU). Informe técnico n. 45 e 46. Campo Grande, MS, 2011.

SILVA, A. M.; SILVA, R. M. da; ALMEIDA, C. A. P. de; CHAVES, J. J. da S. Modelagem geoestatística dos casos de dengue e da variação termopluviométrica em João Pessoa, Brasil. Sociedade \& Natureza, Uberlândia, MG, v. 27, n. 1, p. 157-169, jan./abr. 2015.

TORRES, E. M. Dengue. Tradução de Rogério Dias. Rio de Janeiro: Fiocruz, 2005.

VIANA, D. V.; IGNOTTI, E. A ocorrência da dengue e variações meteorológicas no Brasil: revisão sistemática. Revista Brasileira de Epidemiologia, São Paulo, v. 16, n. 2, p. 240-256, jun. 2013.

WORLD HEALTH ORGANIZATION (WHO). Report on global surveillance of epidemic-prone infectious diseases. WHO/CDS/CSR/ISR/2000.1. World Health Organization, Geneva, 2000. Disponível em: <http://www.who.int/csr/ resources/publications/surveillance/en/WHO_Report_Infectious_Diseases. pdf>. Acesso em: 19 dez. 2016.

YOKOO, S. C. Clima e saúde: estudo sobre a influência do tempo atmosférico no número de notificações de dengue no município de Campo Mourão- PR. In: EPCT - ENCONTRO DE PRODUÇÃO CIENTÍFICA E TECNOLÓGICA, 5., 2010. Anais... Disponível em: <http://www.fecilcam.br/nupem/anais_v_epct/PDF/ ciencias_exatas/19_YOKOO.pdf>. 